\title{
Médiévales
}

Langues, Textes, Histoire

\section{L'exploitation et la gestion des ressources naturelles dans le domaine ducal bourguignon à la fin du XIV siècle}

Uses and Management of Natural Resources into Ducal Properties in Burgundy (late 14th Century)

\section{Corinne Beck et Patrice Beck}

\section{(2) OpenEdition}

\section{Journals}

Édition électronique

URL : https://journals.openedition.org/medievales/3863

DOI : $10.4000 /$ medievales.3863

ISSN : $1777-5892$

Éditeur

Presses universitaires de Vincennes

Édition imprimée

Date de publication : 1 décembre 2007

Pagination : $93-108$

ISBN : 978-2-84292-211-5

ISSN : 0751-2708

\section{Référence électronique}

Corinne Beck et Patrice Beck, «L'exploitation et la gestion des ressources naturelles dans le domaine ducal bourguignon à la fin du xiv siècle », Médiévales [En ligne], 53 | automne 2007, mis en ligne le 02 novembre 2010, consulté le 22 avril 2022. URL : http://journals.openedition.org/medievales/3863 ; DOI : https://doi.org/10.4000/medievales.3863 
Corinne BECK

Patrice BECK

\section{L'EXPLOITATION ET LA GESTION DES RESSOURCES NATURELLES DANS LE DOMAINE DUCAL BOURGUIGNON À LA FIN DU XIV ${ }^{\mathrm{e}}$ SIÈCLE}

En Bourgogne à la fin du Moyen Âge, la seigneurie ducale compte une cinquantaine de domaines. Ils sont peu ou prou équipés non seulement d'installations fonctionnelles utiles à l'exploitation directe ou indirecte des propriétés foncières (fermes, granges, celliers, moulins, pêcheries), mais aussi des outils et des symboles de la puissance publique (fortifications, locaux administratifs et judiciaires), ainsi que des aménagements résidentiels, certes plus ou moins développés et susceptibles d'accueillir à l'occasion le duc, sa famille et sa cour (fig. 1).

Ce parc immobilier constitue un chantier de construction, de rénovation et d'entretien constamment ouvert, donc un prolixe marché de travail et d'exploitation de matériaux; notamment sous Philippe Le Hardi et Marguerite de Flandre, les premiers Valois dont le règne, entre 1364 et 1404, se distingue par la densité et la qualité des opérations de réorganisation et de mise au goût du jour de l'héritage capétien. Ces travaux nourrissent alors le chapitre le plus dispendieux de la gestion du domaine, oscillant entre 79 et 86,5\% des rentrées domaniales annuelles ${ }^{1}$.

En témoigne une documentation comptable d'une très grande richesse comportant plusieurs ensembles emboîtés : les comptabilités des châtellenies, celles des bailliages et celles de la Recette générale qui constitue l'organisme central de décision et de contrôle. Les séries les plus précoces courent depuis les années 1330 et les plus longues au moins jusqu'au milieu du $\mathrm{XVI}^{\mathrm{e}}$ siècle, jusqu'au moment où les gouverneurs royaux abandonnent largement la régie directe.

1. J. RAUZIER, Finances et gestion d'une principauté au XIV siècle. Le duché de Bourgogne (1364-1384), Paris, 1996, p. 606. 


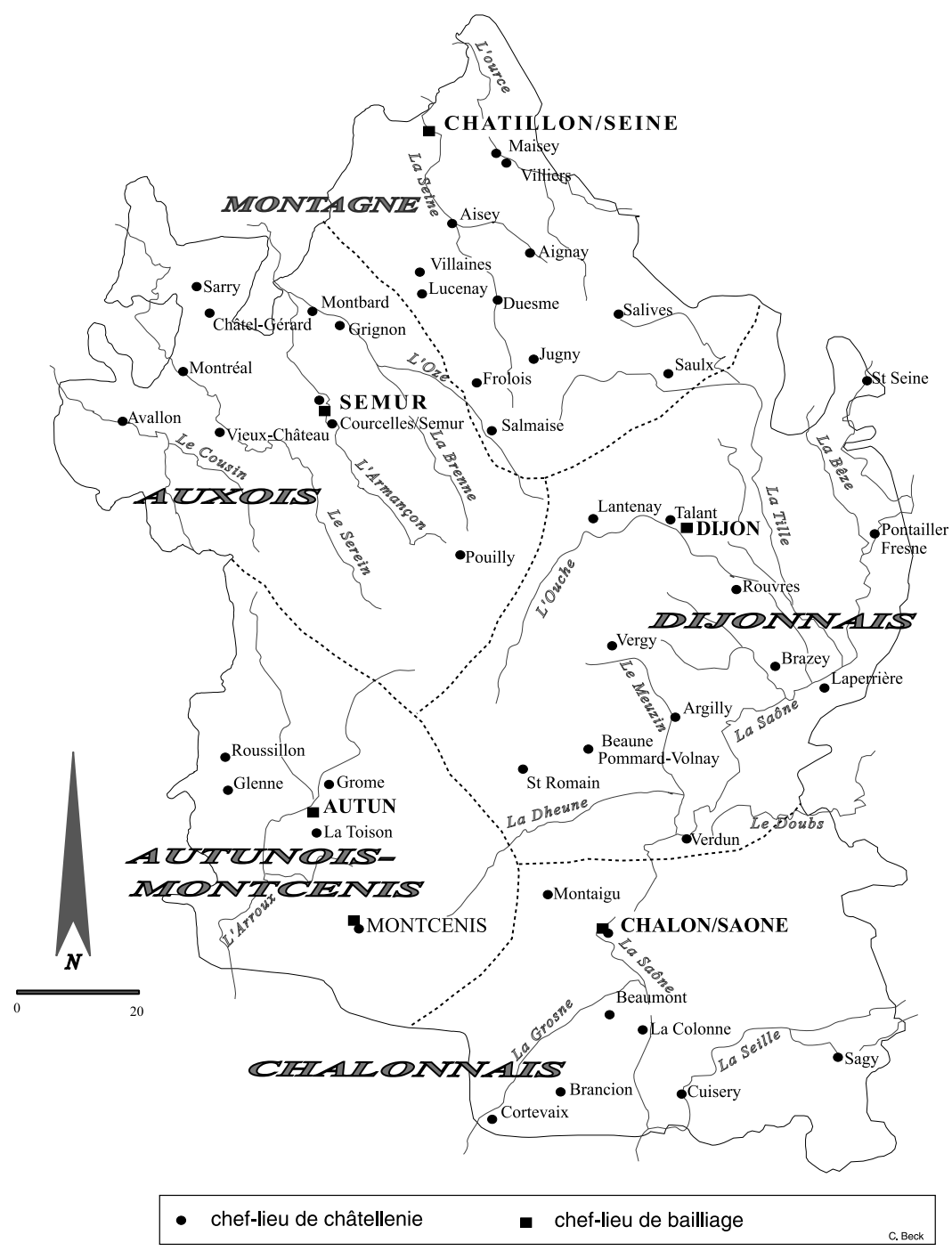

Fig. 1 : Les bailliages et les châtellenies au duché de Bourgogne au début du règne de Philippe le Hardi.

À s'en tenir à trois exemples, la châtellenie d'Aisey dans la vallée de la Seine, le cellier de Chenôve près de Dijon dépendant à la châtellenie de Lantenay, le domaine de Germolles détaché dans les années 1380 de la châtellenie de Montaigu aux portes de Chalon-sur-Saône, ce sont 170 registres de comptabilités, plus de 19300 folios, près de 38600 pages qui sont disponibles. 


\begin{tabular}{|c|c|c|}
\hline AISEY & CHENÔVE & GERMOLLES \\
\hline B 2074 (1328) & B 4262 (1352) & B 4781 (1385) \\
B 2122 (1577) & B 4343 (1663) & B 4822 (1572) \\
\hline 48 registres & 81 registres & 41 registres \\
4 793 folios & 11 499 folios & 3 055 folios \\
\hline
\end{tabular}

Ces comptabilités sont d'excellents observatoires de la vie économique et sociale de ces grands domaines, notamment des usages et des modes de gestions de leurs ressources naturelles: de l'eau et du bois, de la pierre, de la terre et des minéraux qui s'y trouvent et qui y sont exploités. Et les vestiges de ces installations ne sont pas rares : la topographie, le parcellaire et les monuments en conservent de larges traces qui permettent de combiner approches scripturaires et archéologiques.

Dans quelles mesures alors ces domaines fonctionnent-ils avec leurs moyens propres et dans quelles mesures sont-ils conçus comme les pôles d'une même entreprise organisée au niveau de l'État bourguignon ? Quelle est la part respective du domanial et du commercial, voire du spéculatif dans la gestion de leurs ressources naturelles ? Leur économie est-elle de prédation ou bien de développement durable ? Y a-t-il quelque évolution sensible dans les comportements?

Le thème est vaste, impose de brasser un fonds documentaire d'une extrême richesse et si aucune étude d'ensemble n'a été en la matière tentée à ce jour, le dossier comporte désormais de solides enquêtes. Les chantiers de construction et donc leur approvisionnement en matières premières ont été de manière générale explorés par Odette Chapelot dans sa thèse de troisième cycle $^{2}$, puis dans les travaux que nous avons menés sur les aménagements ducaux d'Aisey, Chenôve, Argilly ou Germolles ${ }^{3}$. Les lieux de production des terres cuites architecturales, leur inventaire certes plus que les sites proprement dit, ont aussi été bien explorés par Odette Chapelot qui a donné le premier dénombrement des ateliers ${ }^{4}$; d'autres chercheurs ont poursuivi

2. O. Сhapelot, Les matériaux de la construction en Bourgogne, 1340-1475, aspects techniques et économiques, Thèse de $3^{\mathrm{e}}$ cycle, Paris, 1975.

3. P. BECK, «Un chantier de construction en Châtillonnais à la fin du XIV siècle. Aiseysur-Seine (Côte d'Or), 1389-1391», Annales de Bourgogne, 65, 1993, p. 85-103 ; P. BECK et alii, Le clos de Chenôve. La cuverie et les pressoirs des ducs de Bourgogne. Histoire, archéologie, ethnologie (XIII ${ }^{e}-X^{e}$ s.), Dijon, 1999 ; P. BECK, « De l'atelier au château : production et consommation de briques en Bourgogne au XIV siècle, l'exemple de la châtellenie d'Argilly » dans La brique antique et médiévale, production et commercialisation d'un matériau, Rome, 2000, p. 357-370 ; P. Beck dir., Vie de cour en Bourgogne à la fin du Moyen Âge. Marguerite de Flandre et Germolles (1380-1405), Saint-Cyr-sur-Loire, 2002.

4. O. Сhapelot, Les matériaux..., op. cit.; EAD, «La tuilerie de Montcenis », Milieux, 3-4, oct. 1980, p. 29-39; EAD, «Les tuileries ducales en Bourgogne ( $\mathrm{XIV}^{\mathrm{e}}-\mathrm{XV} \mathrm{e}^{\mathrm{e}} \mathrm{s}$.). L'apport des textes à la connaissance des bâtiments » dans D. Deroeux éd., Terres cuites architecturales au Moyen Âge, Arras, 1986, p. 23-32. 
l'enquête, tel Fabrice Charlier qui a réalisé la fouille archéologique de l'une de ces tuileries, certes seulement de l'atelier et non pas des carrières d'extraction de la matière première ${ }^{5}$. Le fer de l'Auxois, du Châtillonnais et de la Forêt d'Othe est bien connu par les travaux de Michel Mangin ${ }^{6}$, de Paul Benoît ${ }^{7}$, de Catherine Verna ${ }^{8}$, par ceux aussi que nous avons conduits à la fois dans les archives et sur le terrain avec Philippe Braunstein et Michel Philippe $^{9}$. Pour les Eaux et Forêts, notre thèse récente ${ }^{10}$ fait le point sur l'ensemble du domaine ducal bourguignon (fig. 2).

Il y a là une abondante matière à réflexion dont le présent travail voudrait témoigner: en présentant quelques exemples de chantiers transformant les ressources naturelles en matières premières; en énonçant ensuite quelques hypothèses sinon quelques certitudes sur les modes de gestion ainsi déployées, plus particulièrement pour les ressources forestières.

\section{Les ressources des chantiers : nature et lieux du prélèvement}

\section{À Aisey-le-Duc, la reconstruction d'une grange en 1389-1390}

Dans cette localité située à $18 \mathrm{~km}$ en amont de Châtillon dans la haute vallée de la Seine, le châtelain et le maître des œuvres de charpenterie du duc de Bourgogne lancent au cours de l'hiver 1389-1390 des appels d'offre et délivrent des marchés en tâche pour la reconstruction de la grange-étable de la basse-cour du château ${ }^{11}$. Les travaux sont menés sur 21 mois et deux années comptables, de mars 1390 à novembre 1391. Ils coûtent un peu moins de 220 francs, couverts à $55 \%$ par les recettes propres de la châtellenie, à

5. F. Charlier, «Soirans-Fouffrans, La tuilerie (Côte-d'Or): un atelier de terre cuite (XV'-XVII ${ }^{\mathrm{e}}$ s.) », Archéologie médiévale, 24, 1994, p. 301-366.

6. M. MANGIN et alii, Mines et métallurgie chez les Éduens. Le district sidérurgique antique et médiéval du Morvan-Auxois, Besançon, 1992.

7. P. BENoÎT, «La sidérurgie du Châtillonnais après l'avènement du procédé indirect (ca 1480-ca 1570) : matériaux et hypothèses » dans Mines, carrières, métallurgie dans la France médiévale, Paris, 1983, p. 77-116.

8. C. VERNA, «La sidérurgie cistercienne en Champagne méridionale et en Bourgogne du Nord $\left(\mathrm{XII}^{\mathrm{e}}-\mathrm{XV}^{\mathrm{e}} \mathrm{s}\right.$.) » dans L'économie cistercienne. Géographie. Mutations du Moyen Âge aux Temps Modernes, Auch, 1983, p. 207-212.

9. Ph. Braunstein, «Les forges champenoises de la comtesse de Flandre (1372-1404)», Annales ESC, 4, 1987, p. 747-777 ; P. Beck, Ph. Braunstein, M. PhilipPe \& Ch. Dunikovski, "La sidérurgie ancienne en forêt d'Othe ", Proto industries et histoire des forêts, Les cahiers de L'Isard, Toulouse, 1992, p. 301 ; P. BeCK, Ph. Braunstein, M. PhilipPe, « Le bois, le fer et l'eau en forêt d'Othe à la fin du Moyen Âge : bilan et perspectives », Cahiers du centre de Recherches Historiques, 9, 1992, p. 1-13.

10. C. BECK, Société et biodiversité. Les eaux et forêts en Bourgogne ducale (vers 1350vers 1480), thèse de Doctorat d'État, Université de Paris VIII, 2004, à paraître aux Presses Universitaires de Vincennes.

11. P. BECK, «Un chantier de construction en Châtillonnais... », op. cit.; voir aussi C. et P. BECK, «La nature aménagée. Le parc du château d'Aisey-sur-Seine (Bourgogne - XIVe-XVI ${ }^{\mathrm{e}} \mathrm{s}$.) » dans L'homme et la nature au Moyen Âge, Paris, 1996, p. 22-29. 


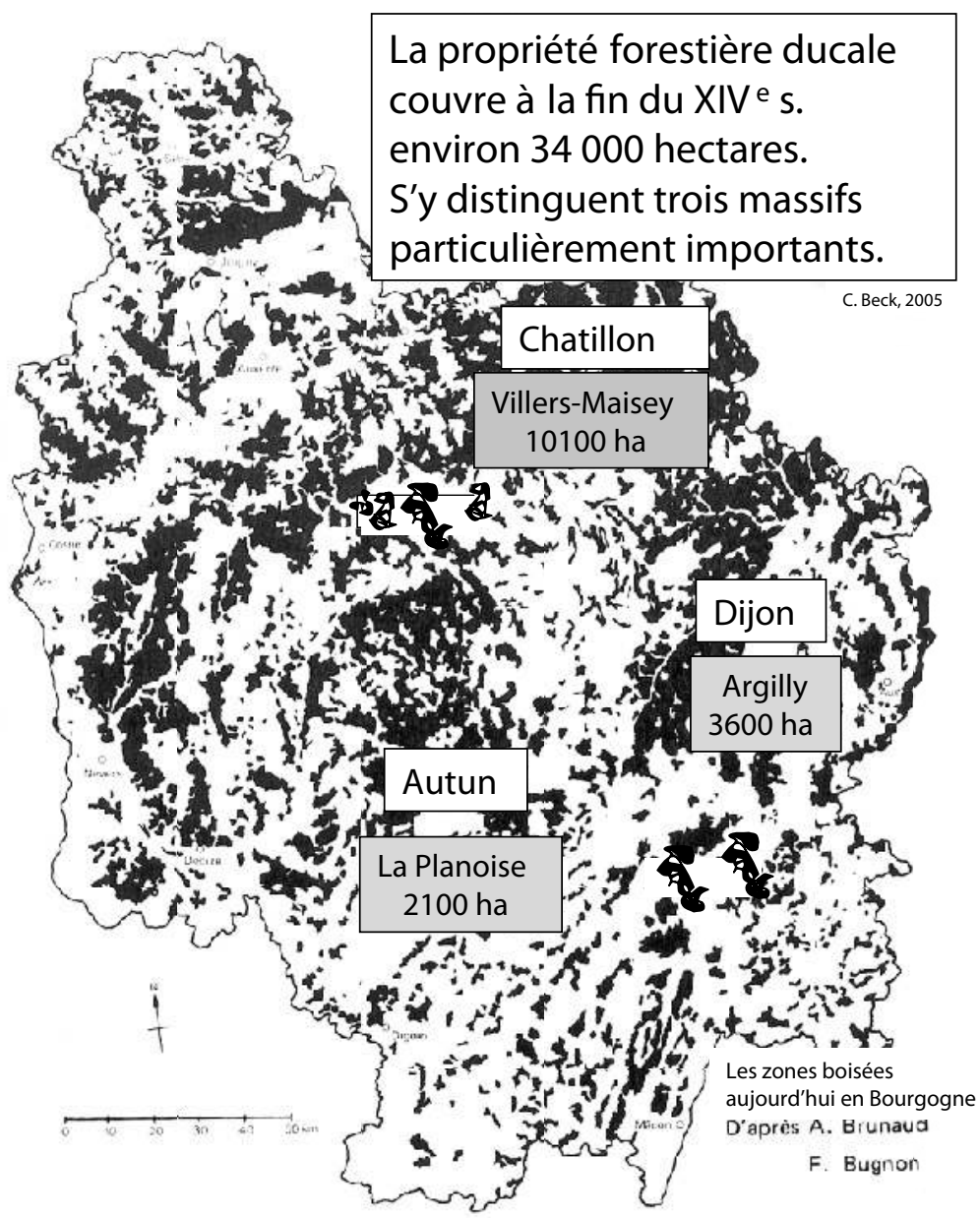

Fig. 2 : Les forêts en Bourgogne, la part ducale,

d'après C. Bonneton éd. Bourgogne, Le Puy-en-Velay, 1985, p. 293.

$45 \%$ par une subvention de 100 francs débloquée le 26 juillet 1390 par le receveur général sur mandement ducal ${ }^{12}$. La gestion du parc immobilier princier, même à ce niveau modeste, procède bien d'une organisation administrative et financière centralisée.

Quant à l'approvisionnement du chantier en matériaux, tuiles, bois de charpenterie et de menuiserie, pierres, terre et chaux proviennent des forêts, 
des carrières et des ateliers les plus proches. C'est même le domaine propre du duc qui fournit l'essentiel et c'est le châtelain qui, le plus souvent, approvisionne et livre le chantier, via son charreton ou les corvées dues. Il est vrai qu'un cloutier et que le serrurier travaillent leur «propre fer », que des laves pour couvrir le bâtiment sont achetées à un particulier. Mais ce sont les seuls cas d'approvisionnement explicitement exogènes car les achats de tuiles à Saint-Marc, dans la même vallée à $4 \mathrm{~km}$ en amont, et de clous à Troyes, à $80 \mathrm{~km}$ en aval, ne font pas exception : les premières sont prises au locataire de la tuilerie ducale la plus proche et, si pour les seconds la certitude n'est pas acquise, il est fort probable qu'en s'approvisionnant à Troyes plutôt que dans les cités voisines de Châtillon et de Montbard, le châtelain continue d'utiliser les ressources domaniales de son employeur qui est producteur de fer en ses forges de la Forêt d'Othe située aux portes de Troyes ${ }^{13}$.

\section{À Chenôve en 1400-1404, la reconstruction des pressoirs}

De cet établissement viti/vinicole installé aux portes de Dijon depuis au moins $1238^{14}$, il est nécessaire en 1398-99 d'étayer le bâtiment qui est « en aventure de cheoir, tellement que l'on n'osait y faire les vendanges ${ }^{15} »$ : la décision est alors prise de tout reconstruire et 14 feuillets de parchemin conservent le détail des travaux qui se déroulent sur quatre années, entre 1400 et $1404{ }^{16}$. Depuis, le bâtiment a perdu sa couverture de laves mais il n'a par ailleurs guère bougé : les bois de sa charpente actuelle, récemment soumis à une analyse dendro-chronologique, ont été coupés au cours de l'hiver $1400^{17}$.

Le coût de l'opération avait été estimé à 1000 francs environ mais il fallut bien plus : 1800 francs, soit une trentaine d'années du salaire journalier d'un maçon ou d'un charpentier ayant travaillé au chantier. Cette somme, huit fois plus importante qu'à Aisey, fut intégralement couverte par des subventions venant de la recette ducale et le chantier, plus complexe que celui d'Aisey, mit à contribution des prestataires recrutés jusqu'à $40 \mathrm{~km}$ autour du chantier. Mais si nombre de corps de métiers - de la pierre, du fer, les cordiers et les vanniers - ont travaillé leur propre matière première dont les documents ne précisent aucunement la provenance, les éléments principaux viennent toujours pour l'essentiel du domaine ducal, proche comme éloigné : les « 14 grosses colonnes et 242 pièces tant tirants, longes et longères, sellières et sablières, comme chevrons et autres pièces à découper [...]» constituant la charpente sont toutes prises «au bois de La Bauche en la châtellenie de La Perrière », c'est-à-dire Outre Saône à une quarantaine de kilomètres du chantier. Il en coûta 242 francs et demi pour acheminer ce bois sélectionné.

13. Ph. BRaunstein, «Les forges champenoises... », op. cit.

14. ADCO $1 \mathrm{H} 1096$.

15. ADCO B 4268-2, fo 42 .

16. ADCO B 4270-6.

17. Le clos de Chenôve..., op. cit. 
La gestion des ressources naturelles comme matières premières des chantiers est bien assurée au niveau central, justement par le corps des « maîtres des œuvres de monseigneur le duc » qui ont compétence pour visiter et maintenir en état tout le parc immobilier ducal et qui doivent bien connaître les ressources et les disponibilités du domaine dans son ensemble.

\section{De la maison-forte à la résidence ducale : les travaux à Germolles} (1382-1392)

Le domaine de Germolles est une acquisition tardive réalisée par Philippe le Hardi en 1380. Il est aussitôt offert à la duchesse Marguerite de Flandre qui y entreprend de grands travaux. Il s'agit de transformer la maison-forte préexistante en une résidence princière dont certains aménagements sont toujours visibles : les analyses dendro-chronologiques réalisées sur les bois de charpente et les lambris du logis actuel font en effet remonter le débitage des premiers à l'automne-hiver 1384-85, celui des seconds entre 1385 et $1405^{18}$. Les travaux sont réalisés par les ateliers des meilleurs artisans et artistes du temps : celui du maître maçon Drouet de Dammartin, des sculpteurs Jean de Marville et Claus Sluter, du peintre Jean de Beaumetz. Ce sont ces personnes qui, dans le même temps, s'activent à l'œuvre monumentale majeure de Philippe le Hardi en Bourgogne : la construction et la décoration de la Chartreuse de Champmol près de Dijon, destinée à accueillir les dépouilles de la dynastie ducale.

Ce chantier de réaménagement s'étala sur une dizaine d'années - de 1382 à 1392 - et les travaux réalisés «du mandement de madame la duchesse » ont été largement couverts par l'administration centrale des finances du duché : la Recette Générale a versé plus de 23500 francs. La somme ne peut certes rivaliser avec les 160000 francs qu'aura coûté le chantier contemporain de la Chartreuse mais elle n'est pas négligeable et encore ne comptabiliset-elle pas les nombreuses dépenses éparses soutenues par d'autres institutions sollicitées à l'occasion d'un besoin précis et ponctuel : les bailliages de Chalon et de Dijon ainsi que diverses châtellenies pourvoyeuses en compétences ou en matériaux et prenant en charge les dépenses. 23500 francs, c'est 12 fois plus que le chantier de Chenôve mais les dispositifs techniques sont globalement identiques. La pierre et le bois «tout venant » proviennent de récupération ou sont extraits des carrières et des forêts locales ; c'est à nouveau la châtellenie de Laperrière-sur-Saône, distante de cinquante $\mathrm{km}$, qui livre les gros bois (fig. 3), par la Saône et à grands frais là encore; l'essentiel des pavements et des tuiles, décorés de figures et glaçurés au plomb, viennent des tuileries de la châtellenie d'Argilly éloignées du chantier de quarante à soixante kilomètres (fig. 4).

18. P. BECK dir., Vie de cour en Bourgogne à la fin du Moyen Âge..., op. cit. 


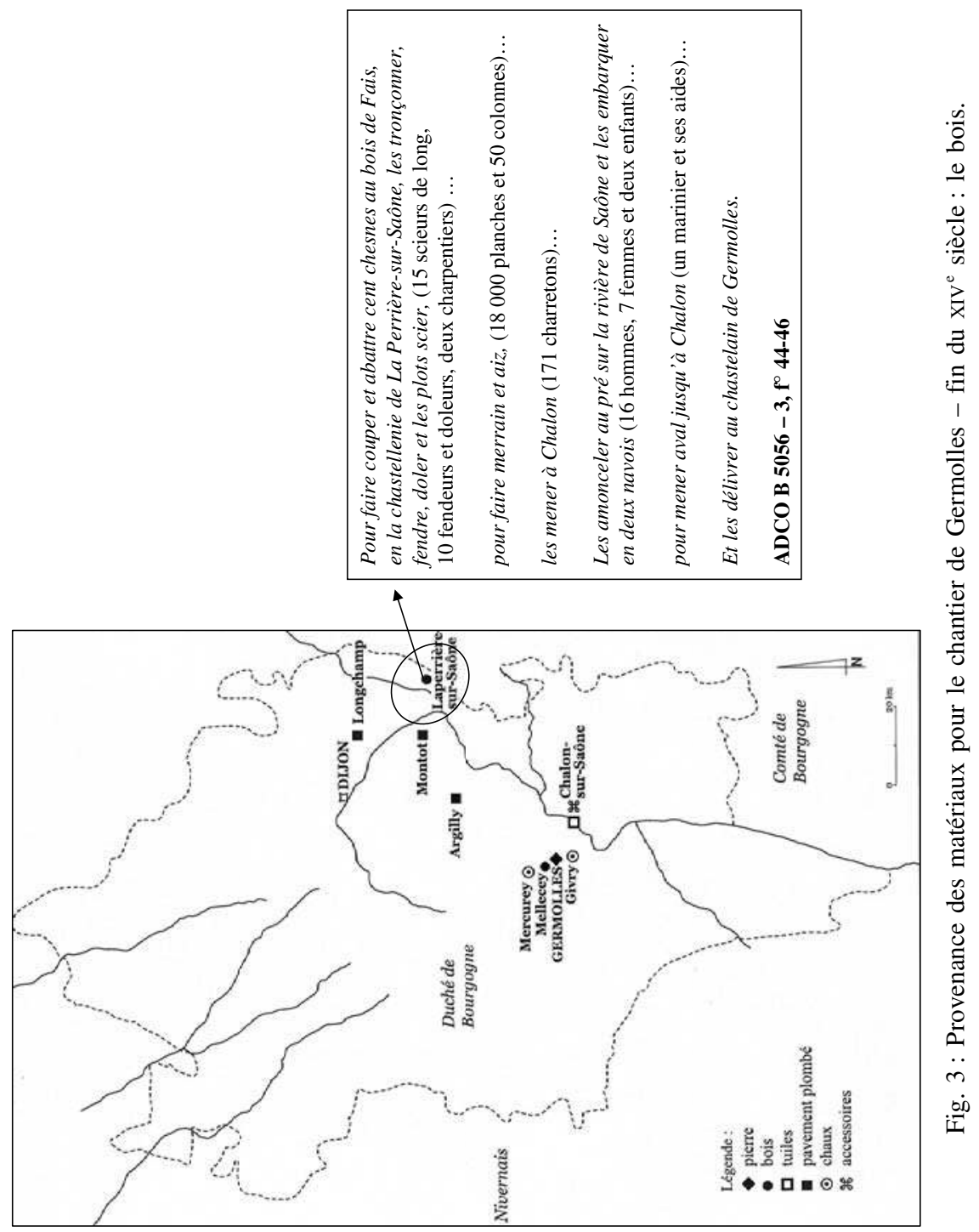




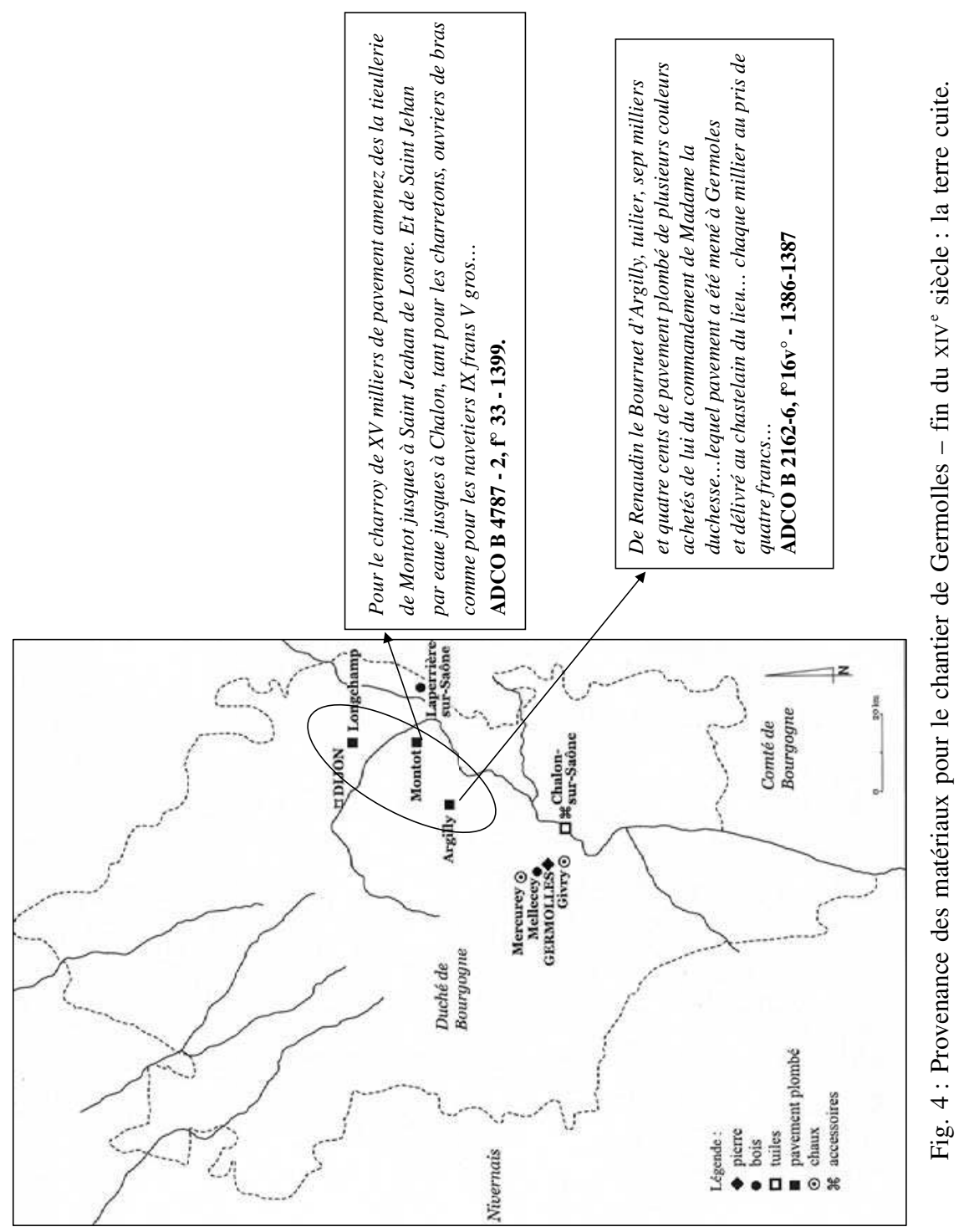




\section{Les entreprises : organisation et importance du prélèvement}

Partout les ateliers employés sur les chantiers ducaux sont nombreux et bien immergés dans le réseau de peuplement rural. Sans doute certains métiers, mettant en œuvre des savoir-faire plus sophistiqués et des matériaux moins courants ou absents des ressources locales, notamment de la serrurerie, de la plomberie et de la verrerie, sont-ils urbains : au plus proche tant des foires et des marchés nécessaires à l'acquisition des marchandises que de la concentration de la clientèle ecclésiastique, aristocratique et bourgeoise. Mais pour les métiers mettant en œuvre les matériaux communs, les artisans sont plutôt attachés aux lieux d'approvisionnement en matières premières. Car l'artisan met souvent en œuvre la matière qu'il a lui-même acquise, choisie puis débitée et façonnée, comme l'indique très clairement la documentation. Le disent les contrats d'achat de cantons ou de pieds de bois et les contrats d'affermage de carrière ou de « minerois », c'est-à-dire du droit de prospection et d'extraction du minerai de fer ; l'expliquent aussi les contrats d'embauche «à la tache » qui précisent souvent que l'artisan aura directement accès aux carrières et aux forêts de son client. Le maçon est aussi carrier, le charpentier aussi bûcheron, le forgeron aussi réducteur du minerai qu'il a lui-même extrait. Mais ces entreprises se révèlent pour la plupart incapables d'assumer seules dans leur spécialité un chantier sortant un peu de l'ordinaire, si bien que plusieurs sources d'approvisionnement et plusieurs ateliers sont généralement associés sur le même chantier. Car la quantité de matériaux employés au cours d'une seule campagne peut être importante et les entreprises artisanales qui s'activent sur les chantiers sont toutes de la dimension «familiale » qu'en Bourgogne et ailleurs on leur reconnaît généralement: un maître accompagné de quelques valets pour les plus nantis, se faisant aider au coup par coup d'hommes de peine.

Il existe bien un programme de représentation du pouvoir princier piloté au plus haut niveau de l'administration ducale, financé par la Chambre des Comptes, organisé par le corps des «maîtres des œuvres de monseigneur le duc », réalisé par les mêmes ingénieurs et artistes circulant d'un chantier à l'autre : les fonteniers de Lantenay présents aussi bien à Aisey qu'à Salmaise et Champmol ${ }^{19}$; le sculpteur flamand Klaus Sluter s'activant aussi bien sur la statuaire de la chartreuse de Champmol que sur celle de Germolles. L'unité de conception et de réalisation est bien visible dans le détail de certaines maçonneries comme dans le programme iconographique développé sur les carreaux de pavement et les peintures murales ${ }^{20}$.

19. P. BECK, «Fontaines et fontainiers des ducs de Bourgogne», Mélanges de l'École Française de Rome, Moyen Âge, 104, 1992, p. 495-506.

20. P. BECK, «Un prince à la campagne. Les résidences de Philippe le Hardi en Bourgogne », Dossier de l'Art, 107, 2004, p. 78-81; ID., «Les résidences ducales ou l'architecture comme mise en scène du pouvoir» dans Le temps des Princes des fleurs de lys. L'art à la cour de Bourgogne entre 1360 et 1420, catalogue d'exposition, Dijon, mai/sept. 2004, Paris, 2004, p. 137-139. 
Mais même au plus près des grands chantiers, la production et donc le prélèvement sur la matière première ne sont pas concentrés. Ils sont au contraire dispersés en de nombreux lieux et distribués entre de nombreuses entreprises de dimensions mesurées et si le problème de l'impact du prélèvement tant sur le paysage que sur la ressource se pose, ce n'est pas un sujet d'interrogation comptable et il n'apparaît pas dans notre documentation; à l'exception toutefois d'un secteur déjà bien connu à cette époque pour sa sensibilité : la ressource forestière. La superficie considérable des eaux et forêts ducales, l'importance des ressources qu'elles offrent et le nombre, la diversité et l'imbrication extrême de ses usagers et de ses usages, ont obligé à une exploitation plus méthodique de cette part importante du domaine, à une surveillance accrue de cette ressource et, pour ce faire, ont entrainé la création d'une administration spécialisée : la gruerie dont les archives sont bien conservées.

\section{La ressource forestière : une gestion sous haute surveillance pour la meilleure satisfaction des besoins ducaux}

À la fin du Moyen Âge, le chêne et secondairement le hêtre domineraient un peu partout dans l'espace ducal et plus généralement en Bourgogne. Mais la forêt ducale compte aussi d'autres essences, toutes aussi importantes comme tremble et charme, noisetier, frêne, érable, saule, aulne et orme, cerisier, pommier et poirier sauvages, aussi du sapin, ainsi que des châtaigniers rencontrés à Marmagne dans l'Autunois. Dans bien des massifs forestiers se juxtaposent diverses essences et donc différentes qualités de bois, s'offrant par conséquent à divers usages.

Ces bois connaissent deux modes de traitement, renvoyant en fait à deux régimes sylvicoles : l'un est dit de haulte forest, ou haute futaye, l'autre est dit de bois revenans. Le premier est la futaie de grande taille peuplée de chênes et de hêtres de plus de 40 ans, domaine par excellence des pratiques pastorales comme la glandée mais aussi de coupes de bois d'œuvre. Le second est constitué de taillis définis comme composés d'arbres jeunes, de moins de 30 ans, d'un diamètre de $15 \mathrm{~cm}$ au maximum et d'une hauteur d'au plus 10 mètres. Ce sont des bois dits de jeune revenue ou de revenue, exploités en courte révolution : 5-10-15 voire 20 ans, pour servir au chauffage, à l'artisanat ou au charbonnage.

L'administration de la gruerie, au temps de Philippe le Hardi, veille à ce que les bois servent d'abord à satisfaire les besoins ducaux : constructions et réparations des châteaux et des hôtels, des haras, des écuries et des granges, des étangs et des ponts, entretien des jardins et des clos de vignes, chauffage des résidences et alimentation des fours domestiques et artisanaux. Car, quand bien même ces travaux se font par marché passé avec des ouvriers des environs, c'est le duc qui fournit le plus souvent la matière première nécessaire. 
Mais tous les bois coupés et débités pour le service des installations ducales, sont loin d'être complètement utilisés. Ils alimentent alors le marché car l'exploitation des forêts ducales ne fait l'économie d'aucun reste. De ces « gastiz de bois », le Trésor ducal tire profit, vendant tant les pièces débitées et non mises en œuvre que les restes des coupes de bois, c'est-à-dire ce qui subsiste sur les souches après que les tiges principales aient été coupées ${ }^{21}$. Le service du prince entraînait du surplus sinon même du gaspillage de la matière ligneuse sur pied mais l'administration réussissait ainsi à en limiter au moins l'impact pécuniaire par ces ventes.

Cela étant, des ventes directes de bois sur pied apparaissent sur le circuit des opérations marchandes. Mais elles sont en général irrégulièrement distribuées aussi bien dans l'espace que dans le temps et c'est surtout après 1390 , quand le patrimoine immobilier est remis en ordre et quand les séjours ducaux ne se font plus qu'exceptionnels en Bourgogne qu'elles se concentrent. Sous Philippe le Hardi, le service du prince passe avant tout.

\section{Une gestion marchande du bois d'œuvre limitée}

Les ventes de bois d'œuvre du domaine ducal ne sont régulières dans aucun massif : elles sont occasionnelles, selon la demande, selon les besoins financiers ducaux. Elles s'effectuent alors selon deux procédés inégalement représentés.

Elles se font surtout au pied, c'est-à-dire à l'unité, en choisissant les arbres sous la surveillance des forestiers. Bien que les rédacteurs des comptes ne mentionnent jamais le mot, ce type de traitement s'apparente à ce que l'on désignait avant le XVIII ${ }^{\mathrm{e}}$ siècle par le terme de «furetage » : une exploitation par pied d'arbre, au gré des besoins et sans ordre préalable. Sur le plan écologique, la pratique du furetage n'est pas sans avantage : elle permet notamment de ne pas couper trop d'arbres brutalement et, en éclaircissant le couvert, de favoriser la croissance de jeunes arbres condamnés jusque-là à végéter.

Les ventes s'effectuent également mais plus rarement à l'unité de surface (arpent, quartier, «corne») : elles s'apparentent alors à ce qu'on appelle aujourd'hui les coupes «par assiette » dont la contenance et l'emplacement étaient d'avance déterminés par les agents ducaux. Dans l'ensemble, les surfaces mises en coupe sont faibles : de 1/4 d'arpent à 10 arpents (soit de 13,36 ares à 534 ares). Certes, des coupes plus importantes existent : jusqu'à 100 arpents à Villiers-Maisey en 1391-92 mais elles font figure d'exception. On peut penser que, compte tenu du niveau élevé des mises à prix et de la taille générale des entreprises connues, de trop larges surfaces n'auraient sans doute pas attiré suffisamment d'adjudicataires.

21. Des exemples en forêt d'Argilly en 1364-65 (ADCO B 2154-b, fo 2 ) et en 1379-80 (ADCO B 2161-8, $\mathrm{f}^{\mathrm{o}} 19 \mathrm{v}^{\circ}$ ), ou à Montréal en 1380-81 (ADCO B 5410, $\mathrm{f}^{\mathrm{o}} 19 \mathrm{v}^{\mathrm{o}}$ ). 
S'agissait-il de coupe rase ou de coupe avec réserve de baliveaux ? Dans les futaies bourguignonnes, la pratique du balivage n'est pas explicitement attestée mais, dans la mesure où les bois taillis la connaissent, on peut penser qu'il en était de même pour les futaies. La question est d'importance car chacun de ces différents traitements induit des conséquences particulières en terme de peuplement. Les coupes rases ou à blanc créent ce que les forestiers appellent aujourd'hui « un paysage en marches d'escaliers », c'est-à-dire une mosaïque artificielle sans transitions progressives avec homogénéisation des peuplements. Ces derniers sont aussi plus fragiles : la coupe à blanc renforce les écarts thermiques et les jeunes pousses sont alors plus exposées au froid et au gel comme à la chaleur et à la sécheresse.

De ces deux types de ventes, le premier est assurément majoritaire au Moyen Âge: si chaque année ou presque sont réalisées des ventes de bois d'œuvre au détail, par pied d'arbre, les coupes annuelles «par assiette» demeurent occasionnelles dans le duché, et cette situation bourguignonne confirme bien les observations générales de Michel Devèze, selon lequel les coupes annuelles de futaie, communes à l'époque moderne, ne se mettraient en place qu'à partir du Xvi ${ }^{\mathrm{e}}$ siècle $^{22}$.

L'acquisition se fait «au plus offrant et derrenier encherisseur », selon des prix fort variables établis bien évidemment en fonction des lois du marché et de l'importance du lot mais aussi en raison, assurément, de l'espèce et, très certainement, de nombreux critères jugés sur pièce et liés à l'âge et aux différences tant de sol que d'exposition, telles la densité de la matière, la configuration et l'importance des fûts, la quantité de branchage de la couronne. C'est ainsi que, dans les bois de Borne de la châtellenie d'Argilly, sont distingués en 1394-95 deux secteurs : l'un « dessus Grantchamp », au sud entre Ruffey et Reullée non loin de la Bouzaise, où l'arpent est vendu 12 francs, l'autre « de costé le chemin ferrey » non loin de Villy-le-Moutier à l'est, où l'arpent est estimé à 35 francs ${ }^{23}$.

C'est avec précision que les bois vendus sont marqués et délimités, pour éviter tout litige et tout abus car ce sont les acheteurs, artisans et marchands, qui assurent l'abattage, comme l'indique l'absence de toute dépense de ce type dans les comptabilités. Les arbres vendus à l'unité sont "seigniés » avec le marteau frappé des armes de Bourgogne. Les secteurs de bois vendus sont mesurés par l'arpenteur du duc et l'assiette spatiale comme les confronts sont indiqués avec précision : en 1391-92 à Villiers-Maisey, sont vendus « $\mathrm{C}$ arpens de bois es bois de Maisy au bout de l'autre vendue que autre fois y avoit estre faicte ainsi comme elle se comporte dez la combe du Poirier roige jusques a l'orme du chemin qui va de Villers le duc a Chastoillon, ainsi comme il est marqué $»^{24}$.

22. M. Devèze, La vie de la forêt française, Paris, 1961, p. 209.

23. Les prix sont données dans le système «franc »: le franc à 12 gros de 20 deniers chacun.

24. ADCO B 1491, f 14 . 
Au total, en matière d'exploitation du bois d'œuvre, l'administration de la gruerie a utilisé différents modes de traitement : furetage, coupe à blanc ou avec réserve de baliveaux. Mais, entre le furetage qui favorise le maintien de la forêt et la coupe à blanc qui peut aboutir à une dégradation des massifs, c'est le premier mode d'exploitation qui est le plus usité aux XIV et $\mathrm{XV}^{\mathrm{e}}$ siècles et, dans tous les cas, la volonté de contrôler voire de restreindre les prélèvements de bois est manifeste. Pour préserver l'exploitation d'autres ressources et d'autres sources de revenu comme la glandée ? Sans doute. Parce que les forêts se régénèrent mal ? Peut-être et il s'agirait alors d'une mesure conservatoire.

De toute façon le bois est placé sous haute surveillance. On ne peut manquer, en effet, de faire le lien avec la mise sur pied et le développement conjoints de corps d'officiers tout spécialement chargés de la gestion forestière aussi bien en duché qu'en comté ${ }^{25}$ et dans les terres bourguignonnes de Flandres. Il n'est certes pas question de protéger l'environnement : il s'agit explicitement de préserver des réserves de chasse et de matériaux pour maintenir le service du prince, de répondre ensuite au souci économique de tirer le meilleur revenu de l'exploitation du domaine. Mais ces préoccupations ont inévitablement pour souci d'assurer la pérennité et donc le renouvellement de la ressource. Ce «programme » de gestion durable est bien présent sous Philippe le Hardi ; il résiste mal ensuite, avec l'éloignement du prince, aux pressions récurrentes de la conjoncture.

Corinne BEck, Université de Valenciennes et du Hainaut-Cambrésis, Le Mont Houy, 59313 Valenciennes Cedex 9

Patrice Beck, Département d'Histoire Université de Lille 3 - UMR 8529, Domaine universitaire du Pont de Bois, BP 60149, 59653 Villeneuve-d'Ascq Cedex

\section{L'exploitation et la gestion des ressources naturelles dans le domaine ducal bourguignon à la fin du $\mathrm{XIV}^{\mathrm{e}}$ siècle}

Les importantes séries comptables de l'administration ducale constituent d'excellents observatoires de la vie économique et sociale des domaines ducaux, notamment des usages et des modes de gestion de leurs ressources naturelles: de l'eau et du bois, de la pierre, de la terre et des minéraux qui s'y trouvent et y sont exploités. À partir de quelques exemples, il s'agit d'analyser le fonctionnement de ces domaines : à la fois avec leurs moyens propres et comme pôles d'une entreprise diversifiée organisée au niveau de l'État bourguignon.

Bourgogne - domaine ducal - fin $\mathrm{XIV}^{\mathrm{e}}$ siècle - matériaux de construction ressources forestières

25. P. GRESSER, La gruerie du comté de Bourgogne aux XIV et XV siècles, Thèse de doctorat d'État en Histoire, université de Bourgogne, 1994, 2 vol. 


\section{Uses and Management of Natural Resources into Ducal Properties} in Burgundy (late 14th Century)

The substantial accounts of the ducal administration accurately highlight the economic and social life of ducal properties, specially the management methods and the uses of their natural resources : water and wood, stone, soil and minerals which were exploited at that time. These properties need to be studied not only through their own entity yet also as various areas of an organized company at the Burgundian duchy level.

Burgundy - ducal property - late 14th century - building materials, forestry resources 
